\title{
Optimized Overlapping Domain Decomposition: Convergence Proofs
}

\author{
Minh-Binh TRAN ${ }^{1}$
}

\section{Introduction}

During the last two decades many domain decomposition algorithms have been constructed and lot of techniques have been developed to prove the convergence of the algorithms at the continuous level. Among the techniques used to prove the convergence of classical Schwarz algorithms, the first technique is the maximum principle used by Schwarz. Adopting this technique M. Gander and $\mathrm{H}$. Zhao proved a convergence result for $\mathrm{n}$-dimensional linear heat equation in Gander and Zhao [2002]. The second technique is that of the orthogonal projections, used by P. L. Lions in Lions [1988], and his convergence results are for linear Laplace equation and linear Stokes equation. In the same paper, P. L. Lions also proved that the Schwarz sequences for linear elliptic equations are related to classical minimization methods over product spaces and this technique was then used by L. Badea in Badea [1991] for nonlinear monotone elliptic problems. Another technique is the Fourier and Laplace transforms used in the papers Giladi and Keller [2002], Gander and Stuart [1998] for some 1-dimensional evolution equations, with constant coefficients. In Lui [2002], Lui [2001], S. H. Lui used the idea of upper-lower solutions methods to study the convergence problem for some PDEs, with initial guess to be an upper or lower solution of the equations and monotone iterations.

For nonoverlapping optimized Schwarz methods, P. L. Lions in Lions [1989] proposed to use an energy estimate argument to study the convergence of the algorithm. The energy estimate technique was then developed in Benamou and Desprès [1997] for Helmholtz equation and it has then become a very powerful tool to study nonoverlapping problems. J.-H. Kimn in Kimn [2005] proved the convergence of an overlapping optimized Schwarz method for Poisson's equation with Robin boundary data and S. Loisel and D. B.

BCAM, Basque Center for Applied Mathematics

Alameda de Mazarredo, 14 E-48009 Bilbao, Basque Country - Spain tbinh@bcamath.org 
Szyld in Loisel and Szyld [2010] extended the technique of J.-H. Kimn to linear symmetric elliptic equation. Another technique is to use semiclassical analysis, which works for overlapping optimized Schwarz methods with rectangle subdomains, linear advection diffusion equations on the half plane (see Nataf and Nier [1998]).

This paper is devoted to the study of the convergence of Schwarz methods at the continuous level. We give a sketch of the proof of the convergence of optimized Schwarz methods for semilinear parabolic equations, with multiple subdomains. Complete convergence proofs for both classical and optimized Schwarz methods, both semilinear parabolic and elliptic equations, with multiple subdomains could be found in Tran [2012].

\section{Convergence for Semilinear Parabolic Equations}

Consider the following parabolic equation

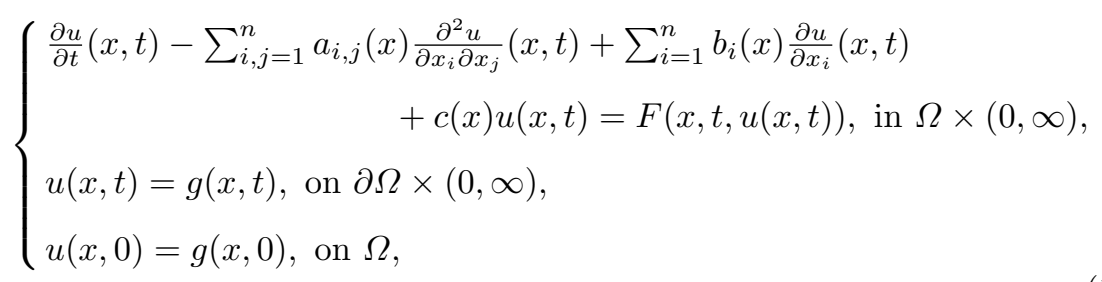

where $\Omega$ is a bounded and smooth enough domain in $\mathbb{R}^{n}$. The following conditions are imposed on (1).

(A1) For all $i, j$ in $\{1, \ldots, I\}, a_{i, j}(x)=a_{j, i}(x)$. There exist strictly positive numbers $\lambda, \Lambda$ such that $A=\left(a_{i, j}(x)\right) \geq \lambda I$ in the sense of symmetric positive definite matrices and $a_{i, j}(x)<\Lambda$ in $\Omega$.

(A2) The functions $a_{i, j}, b_{i}, c$ are in $C^{\infty}\left(\mathbb{R}^{n}\right)$ and $g$ is in $C^{\infty}\left(\mathbb{R}^{n+1}\right)$.

(A3) There exists $C>0$, such that

$\forall t \in \mathbb{R}, \forall x \in \mathbb{R}^{n},\left|F(x, t, z)-F\left(x, t, z^{\prime}\right)\right| \leq C\left|z-z^{\prime}\right|, \forall z, z^{\prime} \in \mathbb{R}$.

We now describe the way that we decompose the domain $\Omega$ : The domain $\Omega$ is divided into $I$ smooth overlapping subdomains $\left\{\Omega_{l}\right\}_{l \in\{1, I\}}$ :

$$
\begin{gathered}
\left(\partial \Omega_{l} \backslash \partial \Omega\right) \cap\left(\partial \Omega_{l^{\prime}} \backslash \partial \Omega\right)=\varnothing, \quad \forall l, l^{\prime} \in\{1, \ldots, I\}, \quad l \neq l^{\prime} ; \\
\forall l \in\{1, \ldots, I\}, \forall l^{\prime}, l^{\prime \prime} \in J_{l}, l^{\prime \prime} \neq l^{\prime}, \quad \Omega_{l^{\prime}} \cap \Omega_{l^{\prime \prime}}=\varnothing,
\end{gathered}
$$

where

$$
\begin{gathered}
J_{l}=\left\{l^{\prime} \mid \Omega_{l^{\prime}} \cap \Omega_{l} \neq \varnothing\right\} ; \\
\cup_{l=1}^{n} \Omega_{l}=\Omega .
\end{gathered}
$$

This decomposition means that we do not consider cross-points in this paper. Denote by $\Gamma_{l, l^{\prime}}$, for $l^{\prime} \in J_{l}$, the set $\left(\partial \Omega_{l} \backslash \partial \Omega\right) \cap \bar{\Omega}_{l^{\prime}}$. The transmission operator 
$\mathfrak{B}_{l, l^{\prime}}$ is of Robin type $\mathfrak{B}_{l, l^{\prime}} v=\sum_{i, j=1}^{n} a_{i, j} \frac{\partial v}{\partial x_{i}} n_{l, l^{\prime}, j}+p_{l, l^{\prime}} v$ and $n_{l, l^{\prime}, j}$ is the $j$-th component of the outward unit normal vector of $\Gamma_{l, l^{\prime}} ; p_{l, l^{\prime}}$ is positive and belongs to $L^{\infty}\left(\Gamma_{l, l^{\prime}}\right)$. The iterate $\# k$ in the $l$-th domain, denoted by $u_{l}^{k}$ of the Schwarz waveform relaxation algorithm is defined by:

$$
\left\{\begin{array}{l}
\frac{\partial u_{l}^{k}}{\partial t}-\sum_{i, j=1}^{n} a_{i, j} \frac{\partial^{2} u_{l}^{k}}{\partial x_{i} \partial x_{j}}+\sum_{i=1}^{n} b_{i} \frac{\partial u_{l}^{k}}{\partial x_{i}}+c u_{l}^{k}=F\left(t, x, u_{l}^{k}\right), \text { in } \Omega_{l} \times(0, \infty), \\
\mathfrak{B}_{l, l^{\prime}} u_{l}^{k}=\mathfrak{B}_{l, l^{\prime}} u_{l^{\prime}}^{k-1}, \text { on } \Gamma_{l, l^{\prime}} \times(0, \infty), \forall l^{\prime} \in J_{l},
\end{array}\right.
$$

where

$$
u_{l}^{k}(x, t)=g(x, t) \text { on }\left(\partial \Omega_{l} \cap \partial \Omega\right) \times(0, \infty), \quad u_{l}^{k}(x, 0)=g(x, 0) \text { in } \Omega_{l} .
$$

The initial guess $u^{0}$ is bounded in $C^{\infty}(\overline{\Omega \times(0, \infty)})$; and at step 0, the Equations (2) is solved with boundary data

$$
\mathfrak{B}_{l, l^{\prime}} u_{l}^{1}(x, t)=u^{0}(x, t) \text { on } \Gamma_{l, l^{\prime}} \times(0, \infty), \forall l^{\prime} \in J_{l} .
$$

A compatibility condition on $u^{0}(x, t)$ is also assumed

$$
\mathfrak{B}_{l, l^{\prime}} g(x, 0)=u^{0}(x, 0) \text { on } \Gamma_{l, l^{\prime}}, \forall l^{\prime} \in J_{l} .
$$

By an induction argument, the algorithm is well-posed. Let $e_{l}^{k}$ be $u_{l}^{k}-u$

$$
\left\{\begin{aligned}
\frac{\partial e_{l}^{k}}{\partial t}-\sum_{i, j=1}^{n} & a_{i, j}(x) \frac{\partial^{2} e_{l}^{k}}{\partial x_{i} \partial x_{j}}+\sum_{i=1}^{n} b_{i}(x) \frac{\partial e_{l}^{k}}{\partial x_{i}} & & \\
& +c(x) e_{l}^{k}=F\left(t, x, u_{l}^{k}\right)-F(t, x, u), & & \text { in } \Omega_{l} \times(0, \infty), \\
\mathfrak{B}_{l, l^{\prime}} e_{l}^{k}(x, t)= & \mathfrak{B}_{l, l^{\prime}} e_{l^{\prime}}^{k-1}(x, t), & & \text { on } \Gamma_{l, l^{\prime}} \times(0, \infty), \forall l^{\prime} \in J_{l} .
\end{aligned}\right.
$$

Moreover,

$$
e_{l}^{k}(x, t)=0 \text { on }\left(\partial \Omega_{l} \cap \partial \Omega\right) \times(0, \infty), \quad e_{l}^{k}(x, 0)=0 \text { in } \Omega_{l} .
$$

For any function $f$ in $L^{2}(0, \infty)$, define

$$
\int_{0}^{\infty} f(x) \exp (-y x) d x .
$$

For any fixed positive number $\alpha$, define

$$
|f|_{\alpha}=\sup _{\alpha^{\prime}>\alpha}\left[\int_{\alpha^{\prime}}^{\alpha^{\prime}+1}\left(\int_{0}^{\infty} f(x) \exp (-y x) d x\right)^{2} d y\right]^{\frac{1}{2}}
$$

and

$$
\mathbb{L}_{\alpha}^{2}(0, \infty)=\left\{f: f \in L^{2}(0, \infty),|f|_{\alpha}<\infty\right\}
$$


Thus $\left(\mathbb{L}_{\alpha}^{2}(0, \infty),|\cdot|_{\alpha}\right)$ is a normed subspace of $L^{2}(0, \infty)$.

Theorem 1. Consider the Schwarz algorithm with Robin transmission conditions and the initial guess $u^{0}$ in $C_{c}^{\infty}(\overline{\Omega \times(0, \infty)})$. There exists a constant $\alpha$ large enough such that

$$
\lim _{k \rightarrow \infty} \sum_{l=1}^{I} \int_{\Omega_{l}}\left|e_{l}^{k}\right|_{\alpha}^{2} d x=0 .
$$

Proof. Let $g_{l}$ be a function bounded and greater than 1 in $C^{\infty}\left(\mathbb{R}^{n}, \mathbb{R}\right), \alpha$ be a positive constant, we define

$$
\Phi_{l}^{k}(x):=\left(\int_{0}^{\infty} e_{l}^{k} \exp (-\alpha t) d t\right) g_{l}(x)
$$

then $\Phi_{l}^{k}(x)$ belongs to $H^{1}\left(\Omega_{l}\right)$.

Let $B_{i}^{l}$ and $C^{l}$ be functions in $L^{\infty}\left(\mathbb{R}^{n}\right)$ defined by

$$
\begin{gathered}
B_{i}^{l}:=b_{i}+\sum_{j=1}^{n}\left(a_{i, j} \frac{\partial_{j} g_{l}}{g_{l}}\right) \\
C^{l}=\left[\frac{\alpha}{2}+\sum_{i, j=1}^{n}\left(-a_{i, j} \frac{2 \partial_{i} g_{l} \partial_{j} g_{l}}{\left(g_{l}\right)^{2}}-\partial_{j} a_{i, j} \frac{\partial_{i} g}{g}+a_{i, j} \frac{\partial_{i, j} g_{l}}{g_{l}}\right)-\sum_{i=1}^{n} b_{i} \frac{\partial_{i} g_{l}}{g_{l}}\right] .
\end{gathered}
$$

Define

$$
\begin{aligned}
\mathfrak{L}_{l R}\left(\Phi_{l}^{k}\right)= & -\sum_{i, j=1}^{n} \partial_{j}\left(a_{i, j} \partial_{i} \Phi_{l}^{k}\right)+\sum_{i=1}^{n} B_{i}^{l} \partial_{i} \Phi_{l}^{k}+C^{l} \Phi_{l}^{k} \\
& +\left\{\int_{0}^{\infty}\left[\left(\frac{\alpha}{2}+c\right) e_{l}^{k}-F\left(u_{l}^{k}\right)+F(u)\right] \exp (-\alpha t) d t\right\} g_{l} .
\end{aligned}
$$

It is possible to suppose $\alpha$ to be large such that $C^{l}$ belongs to $\left(\frac{\alpha}{4}, \alpha\right)$.

Lemma 1. Choose $g_{l}, g_{l^{\prime}}$ such that $\nabla g_{l}=\nabla g_{l^{\prime}}=0$ on $\Gamma_{l, l^{\prime}}$ and $\frac{g_{l^{\prime}}}{g_{l}}>1$ on $\Gamma_{l, l^{\prime}}$, for all $l^{\prime}$ in $J_{l} . \Phi_{l}^{k}$ is then a solution of the following equation

$$
\begin{cases}\mathfrak{L}_{l R}\left(\Phi_{l}^{k}\right)=0, & \text { in } \Omega_{l} \times(0, \infty), \\ \beta_{l} \mathfrak{B}_{l, l^{\prime}}\left(\Phi_{l}^{k}\right)=\mathfrak{B}_{l, l^{\prime}}\left(\Phi_{l^{\prime}}^{k-1}\right) & \text { on } \Gamma_{l, l^{\prime}} \times(0, \infty), \forall l^{\prime} \in J_{l} .\end{cases}
$$

where $\beta_{l}=\frac{g_{l^{\prime}}}{g_{l}}$ on $\Gamma_{l, l^{\prime}}$, for all $l^{\prime}$ in $J_{l}$.

For all $l$ in $\{1, I\}$, denote by $\tilde{\Omega}_{l}$ the open set $\Omega_{l} \backslash \overline{\cup_{l^{\prime} \in J_{l}} \Omega_{l^{\prime}}}$. For all $l$ in $I$ such that $\varphi_{l}^{k+1}=\varphi_{l^{\prime}}^{k}$ on $\Gamma_{l, l^{\prime}}$ for all $l^{\prime}$ in $J_{l}$, let $\varphi_{l}^{k}$ and $\varphi_{l}^{k+1}$ be functions in $H^{1}\left(\tilde{\Omega}_{l}\right)$ and $H^{1}\left(\Omega_{l}\right)$. Use the test functions $\varphi_{l}^{k+1}$ and $\varphi_{l}^{k}$, and take the sum (with respect to $l$ in $\{1, I\})$ of $\int_{\tilde{\Omega}_{l}} \mathfrak{L}_{l R}\left(\Phi_{l}^{k+1}\right) \varphi_{l}^{k+1}$ and $\int_{\tilde{\Omega}_{l}} \mathfrak{L}_{l R}\left(\Phi_{l}^{k}\right) \varphi_{l}^{k}$ to get 


$$
\begin{aligned}
& -\sum_{l=1}^{I}\left\{\int_{\tilde{\Omega}_{l}} C^{l} \Phi_{l}^{k} \varphi_{l}^{k} d x+\right. \\
& +\int_{\tilde{\Omega}_{l}} \sum_{i, j=1}^{n} a_{i, j} \partial_{i} \Phi_{l}^{k} \partial_{j} \varphi_{l}^{k} d x+\sum_{i=1}^{n} \int_{\tilde{\Omega}_{l}} B_{i}^{l} \partial_{i} \Phi_{l}^{k} \varphi_{l}^{k} d x-\sum_{l^{\prime} \in J_{l}} \int_{\Gamma_{l^{\prime}, l}} p_{l^{\prime}, l} \Phi_{l}^{k} \varphi_{l}^{k} d \sigma \\
& \left.+\int_{\tilde{\Omega}_{l}}\left\{\int_{0}^{\infty}\left[\left(\frac{\alpha}{2}+c\right) e_{l}^{k}-F\left(u_{l}^{k}\right)+F(u)\right] \exp (-\alpha t) d t\right\} g_{l} \varphi_{l}^{k} d x\right\} \\
= & \sum_{l=1}^{I} \beta_{l}\left\{\int_{\Omega_{l}} C^{l} \Phi_{l}^{k+1} \varphi_{l}^{k+1} d x+\right. \\
& +\int_{\Omega_{l}} \sum_{i, j=1}^{n} a_{i, j} \partial_{i} \Phi_{l}^{k+1} \partial_{j} \varphi_{l}^{k+1} d x+\sum_{l^{\prime} \in J_{l}} \int_{\Gamma_{l, l^{\prime}}} p_{l, l^{\prime}} \Phi_{l}^{k+1} \varphi_{l}^{k+1} d \sigma \\
& +\int_{\Omega_{l}} \sum_{i=1}^{n} B_{i}^{l} \partial_{i} \Phi_{l}^{k+1} \varphi_{l}^{k+1} d x+ \\
& \left.+\int_{\Omega_{l}}\left\{\int_{0}^{\infty}\left[\left(\frac{\alpha}{2}+c\right) e_{l}^{k+1}-F\left(u_{l}^{k+1}\right)+F(u)\right] \exp (-\alpha t) d t\right\} g_{l} \varphi_{l}^{k+1} d x\right\} .
\end{aligned}
$$

In (5), choose $\varphi_{l}^{k+1}$ to be $\Phi_{l}^{k+1}$, then there exists $\varphi_{l}^{k}$, such that for all $l^{\prime}$ in $J_{l} \varphi_{l}^{k}=\varphi_{l^{\prime}}^{k+1}$ on $\Gamma_{l, l^{\prime}}$ and

$\left\|\varphi_{l}^{k}\right\|_{H^{1}\left(\Omega_{l}\right)} \leq C \sum_{l^{\prime} \in J_{l}}\left\|\varphi_{l^{\prime}}^{k+1}\right\|_{H^{1}\left(\Omega_{l^{\prime}}\right)}$ and $\left\|\varphi_{l}^{k}\right\|_{L^{2}\left(\Omega_{l}\right)} \leq C \sum_{l^{\prime} \in J_{l}}\left\|\varphi_{l^{\prime}}^{k+1}\right\|_{L^{2}\left(\Omega_{l^{\prime}}\right)}$,

where $C$ is a positive constant.

The right hand side of $(5)$ is then greater than or equal to

$$
\begin{aligned}
& \sum_{l=1}^{I} \beta_{l}\left\{\int_{\Omega_{l}} \lambda\left|\nabla \Phi_{l}^{k+1}\right|^{2} d x-\left.\sum_{i=1}^{n} \int_{\Omega_{l}}|| B_{i}^{l}\right|_{L^{\infty}\left(\Omega_{l}\right)}\left|\partial_{i} \Phi_{l}^{k+1}\right|\left|\Phi_{l}^{k+1}\right| d x\right\} . \\
\geq & \sum_{l=1}^{I} \beta_{l}\left\{\int_{\Omega_{l}} \frac{\lambda}{2}\left|\nabla \Phi_{l}^{k+1}\right|^{2} d x+\frac{\alpha}{8} \int_{\Omega_{l}}\left|\Phi_{l}^{k+1}\right|^{2}\right\} .
\end{aligned}
$$

Similarly, the left hand side of (5) is less than or equal to 


$$
\begin{aligned}
& \quad \sum_{l=1}^{I}\left\{\int_{\tilde{\Omega}_{l}} \Lambda\left|\nabla \Phi_{l}^{k}\right|\left|\nabla \varphi_{l}^{k}\right| d x+\sum_{i=1}^{n} \int_{\tilde{\Omega}_{l}}\left\|B_{i}^{l}\right\|_{L^{\infty}\left(\tilde{\Omega}_{l}\right)}\left|\partial_{i} \Phi_{l}^{k}\right|\left|\varphi_{l}^{k}\right| d x\right. \\
& \left.+\sum_{l^{\prime} \in J_{l}}\left\|p_{l^{\prime}, l}\right\|_{L^{\infty}\left(\Gamma_{l^{\prime}, l}\right)}\left(\left\|\Phi_{l}^{k}\right\|_{H^{1}\left(\tilde{\Omega}_{l}\right)}^{2}+\left\|\varphi_{l}^{k}\right\|_{H^{1}\left(\tilde{\Omega}_{l}\right)}^{2}\right)\right\} \\
& \leq \sum_{l=1}^{I} M_{1}\left\{\frac{1}{2}\left(\left\|\nabla \Phi_{l}^{k}\right\|_{L^{2}\left(\tilde{\Omega}_{l}\right)}^{2}+\left(\max _{i \in\{1, I\}}\left\|B_{i}^{l}\right\|_{L^{\infty}\left(\tilde{\Omega}_{l}\right)}\right)^{2}\left\|\varphi_{l}^{k}\right\|_{L^{2}\left(\tilde{\Omega}_{l}\right)}^{2}\right)\right. \\
& \quad+\int_{\tilde{\Omega}_{l}} 2 \alpha\left|\Phi_{l}^{k}\left\|\varphi_{l}^{k}\left|d x+\sum_{l^{\prime} \in J_{l}} \int_{\Gamma_{l^{\prime}, l}} p_{l^{\prime}, l}\right| \Phi_{l}^{k}\right\| \varphi_{l}^{k}\right| d \sigma \\
& \left.\quad+\Lambda\left(\left\|\nabla \Phi_{l}^{k}\right\|_{L^{2}\left(\tilde{\Omega}_{l}\right)}^{2}+\left\|\nabla \varphi_{l}^{k}\right\|_{L^{2}\left(\tilde{\Omega}_{l}\right)}^{2}\right)+\frac{\alpha}{2}\left\|\Phi_{l}^{k}\right\|_{L^{2}\left(\tilde{\Omega}_{l}\right)}^{2}+\frac{\alpha}{2}\left\|\varphi_{l}^{k}\right\|_{L^{2}\left(\tilde{\Omega}_{l}\right)}^{2}\right\}
\end{aligned}
$$

where $M_{1}$ depends only on $\left\{\Omega_{l}\right\}_{l \in\{1, I\}}$ and the equation (3). Choose $\alpha$ such that $\alpha>\left(\max _{i \in\{1, I\}}\left\|B_{i}^{l}\right\|_{L^{\infty}\left(\tilde{\Omega}_{l}\right)}\right)^{2}$, there exists $M_{2}$ positive, depending only on $\left\{\Omega_{l}\right\}_{l \in\{1, I\}}$ and (3) such that the right hand side of (7) is dominated by

$$
\begin{aligned}
& \sum_{l=1}^{I} M_{2}\left\{\int_{\tilde{\Omega}_{l}}\left(\frac{\lambda}{2}\left|\nabla \Phi_{l}^{k}\right|^{2} d x+\frac{\alpha}{8}\left|\Phi_{l}^{k}\right|^{2}+\frac{\lambda}{2}\left|\nabla \Phi_{l}^{k+1}\right|^{2}+\frac{\alpha}{8}\left|\Phi_{l}^{k+1}\right|^{2}\right) d x\right\} \\
\leq & \sum_{l=1}^{I} M_{2}\left(\frac{\lambda}{2}\left\|\nabla \Phi_{l}^{k}\right\|_{L^{2}\left(\Omega_{l}\right)}^{2}+\frac{\alpha}{8}\left\|\Phi_{l}^{k}\right\|_{L^{2}\left(\Omega_{l}\right)}^{2}+\frac{\lambda}{2}\left\|\nabla \Phi_{l}^{k+1}\right\|_{L^{2}\left(\Omega_{l}\right)}^{2}+\frac{\alpha}{8}\left\|\Phi_{l}^{k+1}\right\|_{L^{2}\left(\Omega_{l}\right)}^{2}\right) .
\end{aligned}
$$

Define

$$
E_{k}:=\sum_{l=1}^{I}\left(\frac{\lambda}{2}\left\|\nabla \Phi_{l}^{k}\right\|_{L^{2}\left(\Omega_{l}\right)}^{2}+\frac{\alpha}{8}\left\|\Phi_{l}^{k}\right\|_{L^{2}\left(\Omega_{l}\right)}^{2}\right),
$$

then (6), (7) and (8) imply

$$
\left(\beta-M_{2}\right) E_{k+1} \leq M_{2} E_{k},
$$

where $\beta=\min \left\{\beta_{1}, \ldots, \beta_{I}\right\}$.

Since $M_{2}$ depends only on $\left\{\Omega_{l}\right\}_{l \in\{1, I\}}$ and (3), $\beta$ can be chosen such that

$$
M_{3}:=\frac{M_{2}}{\beta-M_{2}}<1 \text {. }
$$

We get

$$
\begin{aligned}
E_{k} & \leq M_{3}^{k} E_{0} \\
& \leq M_{3}^{k} \sum_{l=1}^{I}\left(\frac{\lambda}{2}\left\|\nabla \Phi_{l}^{0}\right\|_{L^{2}\left(\Omega_{l}\right)}^{2}+\frac{\alpha}{8}\left\|\Phi_{l}^{0}\right\|_{L^{2}\left(\Omega_{l}\right)}^{2}\right) .
\end{aligned}
$$


That deduces

$$
\left\|\Phi_{l}^{k}\right\|_{L^{2}\left(\Omega_{l}\right)}^{2} \leq M_{3}^{k} \sum_{l=1}^{I}\left(\frac{4 \lambda}{\alpha}\left\|\nabla \Phi_{l}^{0}\right\|_{L^{2}\left(\Omega_{l}\right)}^{2}+\left\|\Phi_{l}^{0}\right\|_{L^{2}\left(\Omega_{l}\right)}^{2}\right) .
$$

Since (11) still holds if $M_{3}$ and $\lambda$ are fixed, and $\alpha$ is replaced by $y>\alpha$, then

$$
\begin{aligned}
& \sum_{l=1}^{I} \int_{\Omega_{l}}\left(\int_{0}^{\infty} e_{l}^{k} \exp (-y t) d t g_{l}\right)^{2} d x \\
\leq & M_{3}^{k}\left[\frac{4 \lambda}{y} \sum_{l=1}^{I} \int_{\Omega_{l}}\left(\int_{0}^{\infty}\left|\nabla e_{l}^{0}\right| \exp (-y t) d t\right)^{2} g_{l}^{2} d x\right. \\
& +\frac{4 \lambda}{y} \sum_{l=1}^{I} \int_{\Omega_{l}}\left(\int_{0}^{\infty} e_{l}^{0} \exp (-y t) d t\right)^{2}\left|\nabla g_{l}\right|^{2} d x \\
& \left.+\sum_{l=1}^{I} \int_{\Omega_{l}}\left(\int_{0}^{\infty} e_{l}^{0} \exp (-y t) d t\right)^{2} g_{l}^{2} d x\right] .
\end{aligned}
$$

Let $\alpha^{\prime}$ be a constant larger than or equal to $\alpha,(12)$ implies

$$
\begin{aligned}
& \sum_{l=1}^{I} \int_{\Omega_{l}} \int_{\alpha^{\prime}}^{\alpha^{\prime}+1}\left(\int_{0}^{\infty} e_{l}^{k} \exp (-y t) d t\right)^{2} g_{l}^{2} d y d x \\
\leq & M_{3}^{k}\left[\sum_{l=1}^{I} \int_{\Omega_{l}} \int_{\alpha^{\prime}}^{\alpha^{\prime}+1} \frac{4 \lambda}{y}\left(\int_{0}^{\infty}\left|\nabla e_{l}^{0}\right| \exp (-y t) d t\right)^{2} g_{l}^{2} d y d x\right. \\
& +\sum_{l=1}^{I} \int_{\Omega_{l}} \int_{\alpha^{\prime}}^{\alpha^{\prime}+1} \frac{4 \lambda}{y}\left(\int_{0}^{\infty} e_{l}^{0} \exp (-y t) d t\right)^{2}\left|\nabla g_{l}\right|^{2} d y d x \\
& \left.+\sum_{l=1}^{I} \int_{\Omega_{l}} \int_{\alpha^{\prime}}^{\alpha^{\prime}+1}\left(\int_{0}^{\infty} e_{l}^{0} \exp (-y t) d t\right)^{2} g_{l}^{2} d y d x\right] .
\end{aligned}
$$

Since $u^{0}$ belongs to $C_{c}^{\infty}(\overline{\Omega \times(0, \infty)})$, the right hand side of (13) is bounded by a constant $M_{3}^{k} M_{4}(\alpha)$. The fact that $g_{l}$ is greater than 1 implies

$$
\sum_{l=1}^{I} \int_{\Omega_{l}} \int_{\alpha^{\prime}}^{\alpha^{\prime}+1}\left(\int_{0}^{\infty} e_{l}^{k} \exp (-y t) d t\right)^{2} d y d x \leq M_{3}^{k} M_{4}(\alpha)
$$

Inequality (14) deduces

$$
\lim _{k \rightarrow \infty} \sum_{l=1}^{I} \int_{\Omega_{l}}\left|e_{l}^{k}\right|_{\alpha}^{2} d x=0
$$


Acknowledgements. The author would like to express his gratitude to his thesis advisor, Professor Laurence Halpern for her very kind help and support. He is also indebted to the editor for his kind help. The author has been partially supported by the ERC Advanced Grant FP7-246775 NUMERIWAVES.

\section{References}

Lori Badea. On the Schwarz alternating method with more than two subdomains for nonlinear monotone problems. SIAM J. Numer. Anal., 28(1): 179-204, 1991. ISSN 0036-1429.

Jean-David Benamou and Bruno Desprès. A domain decomposition method for the Helmholtz equation and related optimal control problems. J. Comput. Phys., 136(1):68-82, 1997. ISSN 0021-9991.

Martin J. Gander and Andrew M. Stuart. Space-time continuous analysis of waveform relaxation for the heat equation. SIAM J. Sci. Comput., 19(6): 2014-2031 (electronic), 1998. ISSN 1064-8275.

Martin J. Gander and Hongkai Zhao. Overlapping Schwarz waveform relaxation for the heat equation in $n$ dimensions. BIT, 42(4):779-795, 2002. ISSN 0006-3835.

Eldar Giladi and Herbert B. Keller. Space-time domain decomposition for parabolic problems. Numer. Math., 93(2):279-313, 2002. ISSN 0029-599X.

Jung-Han Kimn. A convergence theory for an overlapping Schwarz algorithm using discontinuous iterates. Numer. Math., 100(1):117-139, 2005. ISSN 0029-599X.

P.-L. Lions. On the Schwarz alternating method. I. In First International Symposium on Domain Decomposition Methods for Partial Differential Equations (Paris, 1987), pages 1-42. SIAM, Philadelphia, PA, 1988.

P.-L. Lions. On the Schwarz alternating method. II. Stochastic interpretation and order properties. In Domain decomposition methods (Los Angeles, CA, 1988), pages 47-70. SIAM, Philadelphia, PA, 1989.

Sébastien Loisel and Daniel B. Szyld. On the geometric convergence of optimized Schwarz methods with applications to elliptic problems. Numer. Math., 114(4):697-728, 2010. ISSN 0029-599X.

S. H. Lui. On linear monotone iteration and Schwarz methods for nonlinear elliptic PDEs. Numer. Math., 93(1):109-129, 2002. ISSN 0029-599X.

Shiu-Hong Lui. On monotone and Schwarz alternating methods for nonlinear elliptic PDEs. M2AN Math. Model. Numer. Anal., 35(1):1-15, 2001. ISSN 0764-583X.

Frédéric Nataf and Francis Nier. Convergence of domain decomposition methods via semi-classical calculus. Comm. Partial Differential Equations, 23 (5-6):1007-1059, 1998. ISSN 0360-5302.

Minh-Binh Tran. Convergence properties of overlapping Schwarz domain decomposition algorithms, submitted. http://arxiv.org/abs/1104.4294, 2012. 